\title{
Dilation of nanonatennas induced by an electromagnetic source
}

\author{
Dominique Barchiesi*, Eric Kremer, Abel Cherouat, \\ Thomas Grosges, Houman Borouchaki
}

\author{
Automatic Mesh Generation and Advanced Methods (GAMMA3 UTT/INRIA) \\ University of Technology of Troyes \\ 12 rue Marie Curie - CS 42060 - FR-10004 Troyes cedex \\ *corresponding author, E-mail: dominique.barchiesi@utt.fr
}

\begin{abstract}
The illumination of plasmonic mesostructures produces high confinement of light in their vicinity. This confinement of light can be enhanced in the gap between the two metallic nanorods of a nanonantenna, in particular for the design of biosensors. The nanometric gap can be reduced if the elevation of temperature of the nanonantenna is sufficient, and therefore the fine tuning of the sensor requires the description of the photo-thermal induced dilation. The multiphysics problem associated to such photo-thermal and mechanical effects is modeled through a finite element method (FEM). The problem consists in computing the electromagnetic field, the temperature and the induced dilation surface. This contribution consists in discussing the numerical efficiencies of a sequential, and a coupled approaches, especially in terms of adaptive meshing of the space of computation. The relationship between the field enhancement and the reduction of the gap is studied. Finally the validity of the $2 \mathrm{D}$ multiphysic model is discussed.
\end{abstract}

\section{Introduction}

The concept of micro-thermic was introduced recently in nanotechnology $[1,2]$. Only a few experimental data on the thermal properties of nano-materials are available $[3,4]$ and a new field of investigation is open, experimentally and theoretically, on the thermal effects at the micro and nanoscale $[5,6,7,8,9]$. A temperature mapping near nanostructures has been proposed recently, by measuring fluorescence anisotropy [4]. The fluorescence lifetime of molecules in the vicinity of metallic nanostructures is known to depends on materials and separation $[10,11]$ but also on temperature [4].

Among the family of nanosensors and nanodevices, the nanoantennas are of great interest in biology [12]. The dimer nanoantenas support multiple resonances and have promising spectral properties [13]. A strong field enhancement appears in the gap of the dimer nanoantenna. This enhancement depends on material and shape $[13,14,15]$. It is straightforward that laser induced effects can lead to non negligible thermal effects in nanostructures [7, 16]. Due to the absorption properties of gold, we can expect a local enhancement of the absorbed power density leading to thermal nanometric source. A rough evaluation of the dilation is following. Assuming an elevation of temperature of
$120 \mathrm{~K}$, the relative dilation of gold is about 0.0018 . Therefore the dilation of a nanoantenna with length $300 \mathrm{~nm}$ can exceed half of a nanometer. The gap between the two gold nanorods being nanometric, this deformation could lead to a non-negligible detuning of the nanoantenna. Moreover, the temperature elevation in the gap could be used to design a nanometric source of heat with applications to modify locally the optical index [17] or to modify the fluorescence rate of molecules [18].

Therefore, we wonder if the temperature of the whole structure can lead to nanometric dilations. The effect of the deformation of metal, induced by its illumination by a laser beam, has never been studied, the thermal studies at this scale being in their infancy. This first 2D study (with infinite $1 \mathrm{D}$ objects along the $x$ direction) is devoted to the comparison of two approaches of multiphysics coupling. The paper is organized as follows. Sec. 2 is devoted to describe the multiphysics model. In Sec. 3, numerical results, applied to nanoantenna are presented and discussed. A study of the influence of the antenna length, gap and polarization of the illumination is proposed. The Sec. 3.3 is devoted to a first validation of the $2 \mathrm{D}$ approach with a $3 \mathrm{D}$ benchmark, before concluding in Sec. 4.

\section{The multiphysics model}

It has been shown that finite element method can be used to compute accurately the field enhancement in nanostructures, provided an adapted method of remeshing [19, 20]. This contribution proposes a finite element method of the multiphysics problem of coupling between electromagnetism, thermics and mechanics, with an adaptive remeshing method. The Galerkin formulation of the finite element method requires to define the boundary conditions on the inner boundaries $\Gamma_{1}$ between materials and on the outer boundary $\Gamma_{0}$, the frontier of the domain of computation. The radius of the circular domain of computation is $R_{e}$.

The geometry is defined in the $(y, z)$ plane and the subscript 1 (resp. 2) indicates the gold nanonatenna (resp. the surrounding medium: air). The inset in Fig. 4 illustrates the geometry. The material electromagnetic, thermic and mechanic characteristics are constants for each material, and therefore the multiphysics problem is linear. 


\subsection{Electromagnetism}

The computation of the electromagnetic field is obtained from the finite element Galerkin formulation of the Helmholtz' equation with complex permittivity $\epsilon_{r}(y, z)$ [19]. The pure imaginary is $\imath$ with $\imath^{2}=-1$. The unknown field is the magnetic $x$ component, the considered polarization of the incoming being $p$. Therefore, the electromagnetic problem is scalar. The incoming wave is monochromatic with angular frequency $\omega$ and wavelength in vacuum $\lambda_{0}$. The wave vector $\mathbf{k}_{\mathbf{0}}$ of the incoming wave is along $z$ and therefore the electric field is along $y,\left|\mathbf{k}_{\mathbf{0}}\right|=k_{0}$. $c$ is the speed of light in vacuum and $\epsilon_{0}$ is the permittivity of vacuum. The material parameter is the relative permittivity $\epsilon_{r}(y, z)$ which is assumed to be a dimensionless constant in each isotropic and homogeneous material. The electromagnetic problem is therefore linear. The partial differential equation (PDE) is

$$
\begin{gathered}
{\left[\nabla \cdot\left(\frac{1}{\epsilon_{r}(y, z)} \nabla\right)+k_{0}^{2}\right] H_{x}(y, z)=0} \\
\mathbf{n}_{12} \cdot\left[\left(\frac{1}{\epsilon_{2}} \nabla H_{2}\right)-\left(\frac{1}{\epsilon_{1}} \nabla H_{1}\right)\right]=0 \text { on } \Gamma_{1} \\
\mathbf{n}_{12} \cdot\left[\left(\frac{1}{\epsilon_{2}} \nabla H_{2}\right)\right]=\imath k_{0} H_{1} \text { on } \Gamma_{0}
\end{gathered}
$$

The natural conditions on the external boundaries are the classical diffusion conditions [21]. The natural boundary condition at the interface between materials is the continuity of the normal component of the electric excitation. Then the electric field is deduced from the magnetic field and the Maxwell-Ampere equation. To compute the dilation, an hypothesis on the illumination is used: a gaussian beam with power $P$, and diameter $D$ [22]. Therefore, the amplitude of the incoming electric field on the external boundary $\Gamma_{0}$ can be deduced [23] :

$$
E_{0}=\sqrt{2 \frac{4 P}{c \epsilon_{0} \pi D^{2}}} e^{-\frac{4 R_{e}^{2}}{D^{2}}}
$$

The absorbed electromagnetic field produces a source term for the stationary heat equation, which is proportional to the imaginary part of the permittivity of media $\epsilon_{0} \epsilon_{r}(y, z)$ :

$$
Q(y, z)=\frac{\omega \epsilon_{0} \Im\left(\epsilon_{r}(y, z)\right)}{2}\left|\mathbf{E}(y, z)\left(\epsilon_{r}\right)\right|^{2}
$$

The following numerical parameters are used in computations. The wavelength is $\lambda_{0}=632.8 \mathrm{~nm}, \epsilon_{1}=(0.2+$ $3.32 \imath)^{2}=-11+1.33 \imath$ (gold), $\epsilon_{2}=1$ (air), $D=25 \mu \mathrm{m}$, $P=30 \mathrm{~mW}$. The thermal source is proportional to the imaginary part of the relative permittivity $\epsilon_{1}$.

\subsection{Thermics}

The stationary heat equation is solved assuming thermal conductivities $\lambda(y, z)$, including the mesoscopic correction related to the hypothesis of phonon diffusion and of electron-phonon interaction $[24,25]$. The temperature variation depends on both imaginary part of the permittivity and the thermal conductivity. The remeshing process enables the control of the numerical error on the temperature [23].

$$
[\nabla \cdot(\lambda(y, z) \nabla)] T(y, z)+Q(y, z)=0
$$

with the following boundary conditions:

$$
\begin{array}{r}
\mathbf{n}_{12} \cdot\left[\left(\lambda_{2} \nabla T_{2}\right)-\left(\lambda_{1} \nabla T_{1}\right)\right]=0 \text { on } \Gamma_{1} \\
\mathbf{n}_{12} \cdot\left(\lambda_{2} \nabla T_{2}\right)=-T_{2} \text { on } \Gamma_{0}
\end{array}
$$

The natural boundary conditions are the flux conservation across the material interfaces and the convective condition at the external boundary [23]. At a convective boundary, the heat loss is proportional to the difference of temperature between the surface and the coolant. In this approach, the temperature elevation is used for the dilation computation and therefore, the temperature of the coolant is arbitrary set to 0 . The thermal source $Q$ exists only in the medium 1 (nanonatenna). The following numerical parameters are used in computations. The thermal conductivity of a nanostructure is reduced in nanowires [26] and depends on their length. It can be evaluated to $125 W . K^{-1} . m^{-1}$ in the considered case, the factor of reduction being about 0.5 . The electron and phonons mean-free path in Gold being about $40 \mathrm{~nm}$, the typical size $r_{c}$ of the nanostructures, the effective thermal conductivity can be evaluated:

$$
\lambda_{1} \approx \frac{\lambda_{b u l k}}{1+\frac{4}{3} \frac{l_{p}}{r_{c}}} \approx \frac{1}{2} \lambda_{b u l k} .
$$

In the numerical applications, the media are supposed to be isotropic and homogenous. The thermal conductivities of bulk gold being $\lambda_{\text {Bulk }}=250 \mathrm{Wm}^{-1} \mathrm{~K}^{-1}$, we therefore use $\lambda_{1}=125 \mathrm{Wm}^{-1} K^{-1}$ and $\lambda_{2}=0.026 \mathrm{Wm}^{-1} K^{-1}$ (air). The computed temperature $T$ is the source for the problem of thermal dilation.

\subsection{Mechanics}

The temperature elevation in the whole domain of computation and its derivative are used to compute the dilation within a classical thermo-elasticity formulation. The dilation are expected to be nanometric and the materials are described within elasticity theory. The constitutive relations, considering orthotropics materials with Lamé constants, where $E$ is the Young's modulus and $\nu$ the Poisson's coefficient, enable to compute the stress and to deduce the displacement $\mathbf{U}=\left(U_{y}, U_{z}\right)$ of the interfaces of materials, assuming isotropic thermal dilatation coefficient $\alpha$. In the following, $G=E /((1+\nu)(1-2 \nu))$, $C_{11}=G(1-\nu)=C_{22}, C_{12}=G \nu, C_{33}=G(1-2 \nu) / 2$ and $b=G \alpha(1+\nu)$.

$$
\begin{aligned}
\partial_{y}\left(C_{11} \partial y U_{y}+C_{12} \partial_{z} U_{z}\right)+ & \\
\partial_{z}\left(C_{33}\left(\partial_{z} U_{y}+\partial_{y} U_{z}\right)\right) & =\partial_{y} b T \\
\partial_{z}\left(C_{12} \partial y U_{y}+C_{22} \partial_{z} U_{z}\right)+ & \\
\partial_{y}\left(C_{33}\left(\partial_{z} U_{y}+\partial_{y} U_{z}\right)\right) & =\partial_{z} b T
\end{aligned}
$$


The natural boundary conditions are deduced from the continuity of the displacement of boundaries. The boundaries are free (there is no load on them). In the numerical applications, the media are supposed to be homogenous. The following numerical parameters are used: $\nu_{1}=0.44$ (gold), $\nu_{2}=0$ (air); $E_{1}=78 \mathrm{GPa}$ (gold), $E_{2}=142 \mathrm{kPa}$ (air); $\alpha_{1}=14.6 \cdot 10^{-6} K^{-1}$ (gold), $\alpha_{2}=1 / 273.15 K^{-1}$ (air).

The multiphysics problem can be solved for an arbitrary geometry by using a finite elements method.

\subsection{The finite element methods}

The Galerkin finite element formulation [21] uses a mesh adaptation to regrid the domain of computation to reach a topology error is lower than $10^{-4}$. Two main approaches of the multiphysics problem can be considered. Both are perturbation methods, assuming small dilations, which do not modify the physical constants (permittivity, thermal conductivity and mechanical characteristics):

- M1: The three problems are considered sequentially: first the electromagnetic field is computed. Then the temperature is computed from the stationary heat equation with the electromagnetic source term. Finally the dilation is computed from the temperature. Each problem is solved independently and therefore the inversion method can be adapted to the symmetry of the coupling matrix: the Lanczos method [27] is used for the electromagnetic problem, and the orthomin [28] method is used for the thermics and dilation problems.

- M2: The three problems are solved together, on a common mesh and the same method of inversion (Lanczos) are used.

The error control and the specificity of each physical problem could lead to the premature conclusion that M1 is more efficient than M2. Actually, the effective coupling of EDP can only be handled by M2. Nevertheless, M1 can be considered as a reference, to control the accuracy of M2.

The precision $p$ is the maximal relative variation of each computed solution, between two adjacent nodes, over the entire domain of computation. The field enhancement in the gap of the nanonatenna requires fine grid [19]. The minimum distance between nodes is $\lambda_{0} / 1000$ to reproduce the high gradient of the electric field in the gap. This fine meshing is not contradictory with the continuous model of matter, as it is required only to reproduce accurately the field variations in the vicinity of the matter. On the contrary, a much less fine grid can be used to compute the temperature as it is quasi homogeneous in the material structure and exhibit lower spatial variations. The temperature being the source of dilations, a similar corse grid can be used for dilation.

A systematic study of the influence of the method of resolution is of interest. Table 1 shows the number of adaptive meshing, the final number of cells and the precision for each problem.
Table 1: Performances of the decoupled (M1) and coupled (M2) methods. $N_{r}$ is the total number of remeshing, the number of cells $N_{c}$ and the precision (maximum of error between adjacent nodes among the whole mesh). For method M1, the final mesh of a problem is not imported in the next one. The computational time is less than $4 \mathrm{~min}$ for both methods.

\begin{tabular}{ccccc}
\hline Method & Problem & $N_{r}$ & $N_{c}$ & $p$ \\
\hline M1 & Electrom. & 2 & 1834 & $1.410^{-3}$ \\
& & 6 & 8676 & $1.310^{-4}$ \\
& & 11 & 39404 & $1.310^{-5}$ \\
& Thermics & 2 & 1592 & $9.410^{-6}$ \\
& & 5 & 2406 & $7.110^{-6}$ \\
& & 9 & 34110 & $1.210^{-6}$ \\
& Mechanics & 1 & 3233 & $8.710^{-4}$ \\
& & 3 & 3585 & $1.510^{-4}$ \\
M2 & & 5 & 9644 & $1.610^{-5}$ \\
& & 9 & 57770 & $1.610^{-6}$ \\
& & 2 & 3233 & $1.410^{-3}$ \\
& & 10 & 4914 & $8.410^{-4}$ \\
& & 12 & 6343 & $3.210^{-4}$ \\
\hline
\end{tabular}

The convergence of the methods is studied as a function of the maximal error on the computed variable (Tab. 1). The three problems does not exhibit the same speed of convergence. The strong electric field enhancement in the gap requires at least 11 remeshing to reach a precision lower than $10^{-4}$. On the contrary, the smooth variations of the temperature are revealed with a corse grid. The numerical resolution of the mechanics problem exhibits the same behavior as the thermic one. If the three problems are solved simultaneously (M2), the precision is close to that of the electromagnetic problem. M2 shows a slower convergence than M1. Therefore, solving the coupled problem is less stable as that of the recursive one (M1). The quality of M1 and M2 should also be compared in term of solutions. Table 2 shows the maxima of each computed unknown over the domain of computation.

Table 2: Convergence for the variables of interest. The incoming field intensity being $\left|E_{0}\right|^{2}=4 \cdot 48 \cdot 10^{10}$. The computations are made for a $10 \mathrm{~nm}$ gap and corresponds to the precisions indicated in Tab. 1.

\begin{tabular}{|c|c|c|c|}
\hline \multicolumn{2}{|c|}{ Electromagnetism } & Thermics & \multirow[t]{2}{*}{ Mechanics } \\
\hline \multicolumn{3}{|c|}{ M1 } & \\
\hline $\max |E|^{2}$ & $\max (Q)$ & $\max (T)$ & $\max (|U|)$ \\
\hline$\left(V \cdot m^{-1}\right)$ & $\left(W \cdot m^{-3}\right)$ & $(K)$ & $(n m)$ \\
\hline $1.4910^{12}$ & $1.25810^{15}$ & 149.3 & 0.6 \\
\hline $1.4610^{12}$ & $1.25510^{15}$ & 149.3 & 0.6 \\
\hline $1.4810^{12}$ & $1.25510^{15}$ & 149.3 & 0.6 \\
\hline \multicolumn{4}{|c|}{ M2 } \\
\hline $1.4910^{12}$ & $1.25610^{15}$ & 149.5 & 0.6 \\
\hline $1.4910^{12}$ & $1.25510^{15}$ & 149.5 & 0.6 \\
\hline $1.4910^{12}$ & $1.25510^{15}$ & 149.5 & 0.6 \\
\hline
\end{tabular}




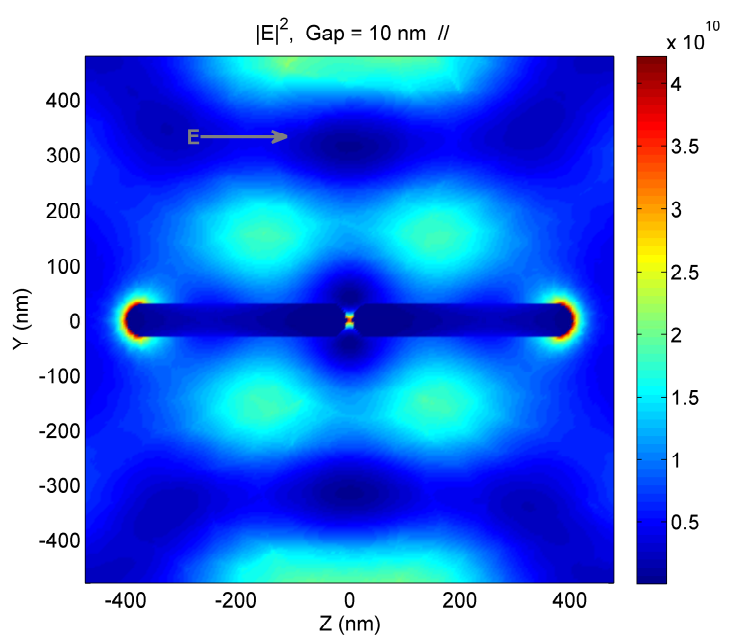

Figure 1: Intensity $|E|^{2}$ showing the high confinement of light in the gap between the gold dimer, $L=400 \mathrm{~nm}, R_{1}=$ $30 \mathrm{~nm}$ and a $10 \mathrm{~nm}$ gap.

The results in Table 2 show that both methods give the same results even if their numerical properties are not the same (Tab. 1). According to the illumination power and focusing, the temperature elevation can overpass $100 \mathrm{~K}$ in the gold structures. The dilation of each nanorod induces a decrease of the gap of more than $10 \%$. These values depend on the optical and thermal constants, and on the illumination properties.

The application of this model to the study of the temperature and dilation as a function of the gap is of interest for practical applications.

\section{The dilation of nanoantenna: the gap modification}

In this section two polarizations of illumination are considered. If the electric field is parallel to the long axis of the nanonatenna, the polarization is called parallel $\|$. The perpendicular polarization $(\perp)$ is also investigated. The purpose is to find a relationship between the field enhancement, the temperature elevation and the dilation. To assess the results, various lengthes $L$ and gaps are used. The radius of the domain of computation is $2 \mu \mathrm{m}$.

\subsection{Polarization $\|$}

Figures 1-4 illustrate the results of computations for gold nanonatenna with $R_{1}=30 \mathrm{~nm}$ and for various lengthes $L$ and gaps.

The electromagnetic intensity (the square of the electric field) is confined in the gap and the finite element method is able to describe this high gradient $[29,20]$. The source of heat $Q$ is proportional to the intensity of the electric field. The temperature elevation $\Delta T$ is quasi uniform in the gold metal, but the dilation is localized at the end of the nanorods and in the gap.

In figure 4 are plotted the dilation for various gaps. The

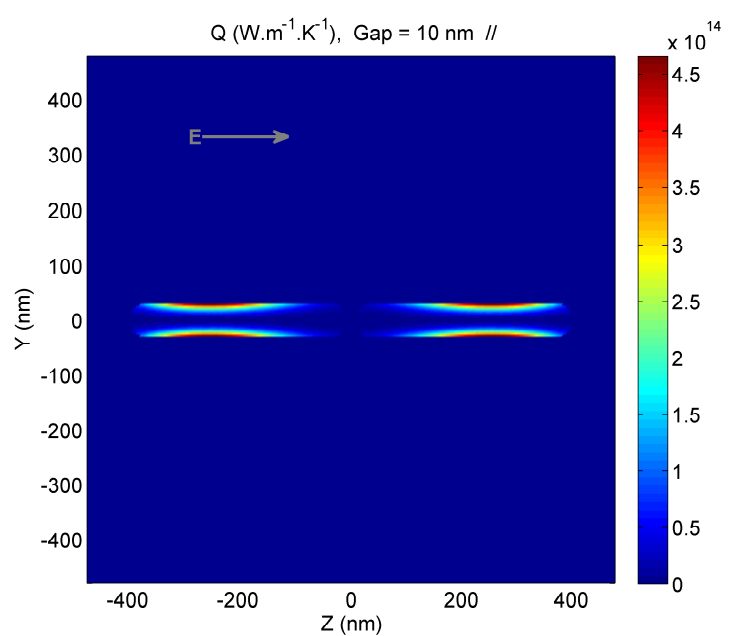

Figure 2: Density of heat source for the stationary heat equation, induced by illumination at $632.8 \mathrm{~nm}$ : a gaussian beam of diameter $25 \mu \mathrm{m}$, with power $P=30 \mathrm{~mW}$. $L=400 \mathrm{~nm}, R_{1}=30 \mathrm{~nm}$ and a $10 \mathrm{~nm}$ gap.

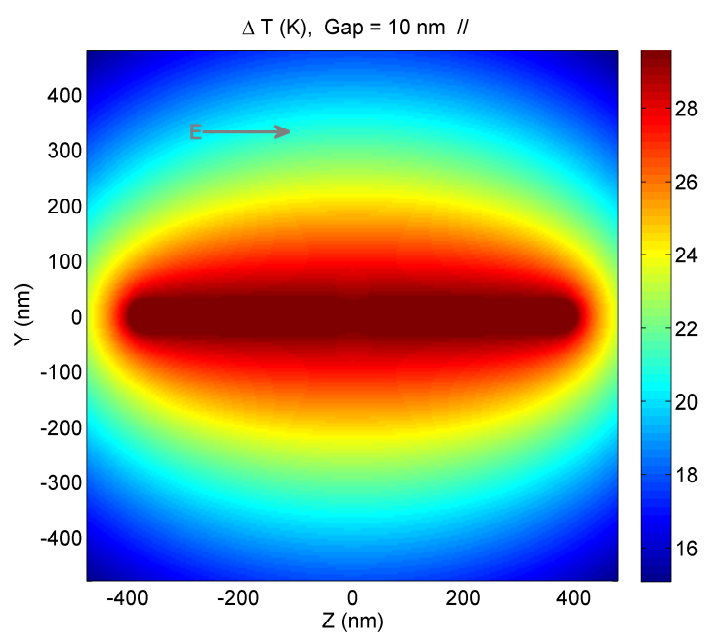

Figure 3: Temperature elevation, solution of the stationary heat equation. $L=400 \mathrm{~nm}, R_{1}=30 \mathrm{~nm}$ and a $10 \mathrm{~nm}$ gap.

dilation appears to be slightly higher in the gap than at the ends of the dimer. The coupling between the two gold particles is reduced for the $200 \mathrm{~nm}$ gap and the field enhancement tends to become identical at their ends and also the relative dilation. The total energy is conserved and therefore, for small gaps, the field enhancement in the gap which contributes to the scattering of light by the nanoantenna, reduces the absorption of energy and the temperature elevation. Figure 5 shows the variation of temperature as a function of the initial gap. If the length of particles is lower than $250 \mathrm{~nm}$, the temperature elevation is governed by the field enhancement in the gap. On the contrary, for larger lengthes, the temperature increases with the gap. Therefore, we could conclude to the existence of two thermal regimes: the long nanoantenna behaves as a single nanoparticle without gap. 


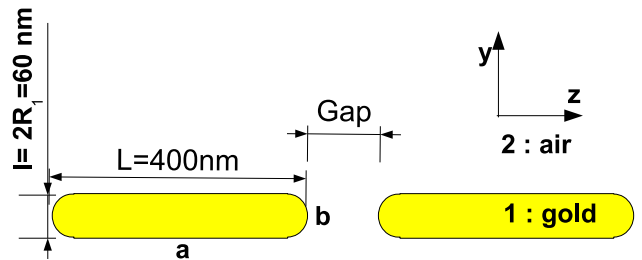

$/ /, \mathrm{L}=400 \mathrm{~nm}$

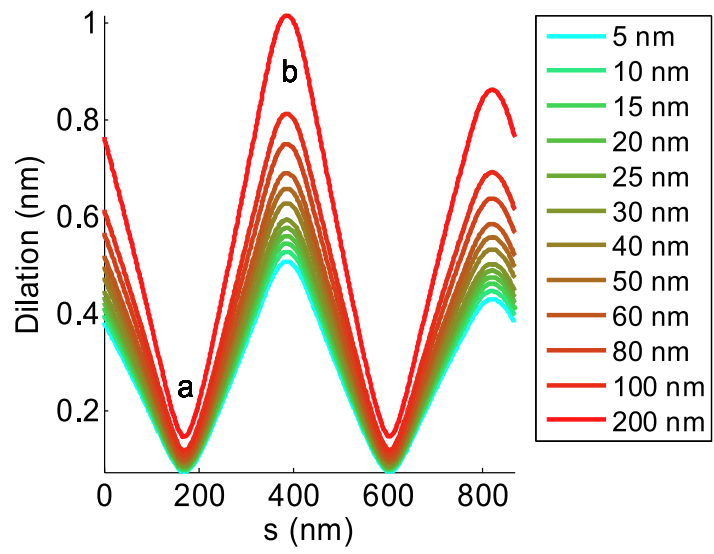

Figure 4: Photo-thermally induced dilations of the surface of each nanorod (the two nanorods are a dimer nanonatenna). $L=400 \mathrm{~nm}, R_{1}=30 \mathrm{~nm}$ and the gap is indicated.

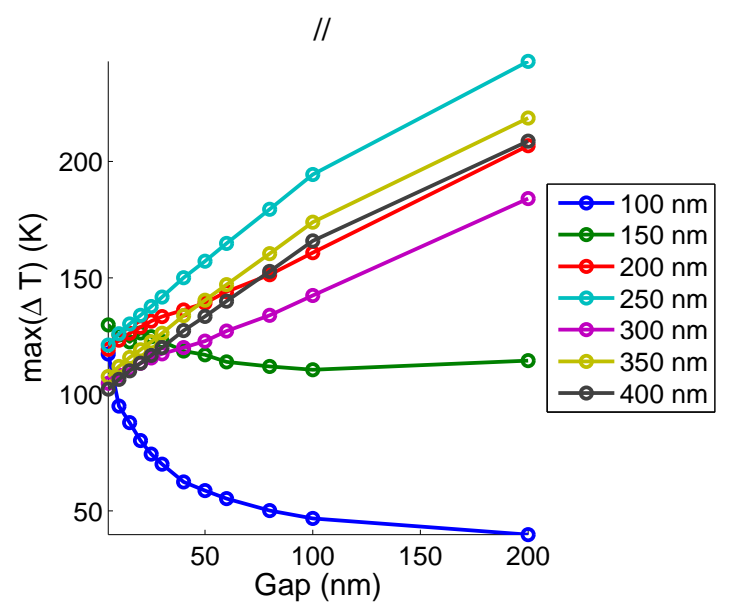

Figure 5: Temperature elevation as a function of the initial gap (in $\mathrm{nm}$ ).

The figure 6 shows the gap reduction in percent under the above described illumination. The reduction of the smaller gap $(10 \mathrm{~nm})$ is about $10 \%$ and therefore for long nanoantenna. For small gaps, the absolute relative reduction increases rapidly with the length of particles and tends toward a limit when the gap reaches $200 \mathrm{~nm}$. Consequently, the volume of the nanoantenna which increases the integral of the thermal source $Q$ should be considered for the analysis of the relative gap reduction.

The dilation of nanonantenna is not linearly connected to the temperature increase with the gap. The intensity enhancement (the ratio of the maximum of intensity in the gap

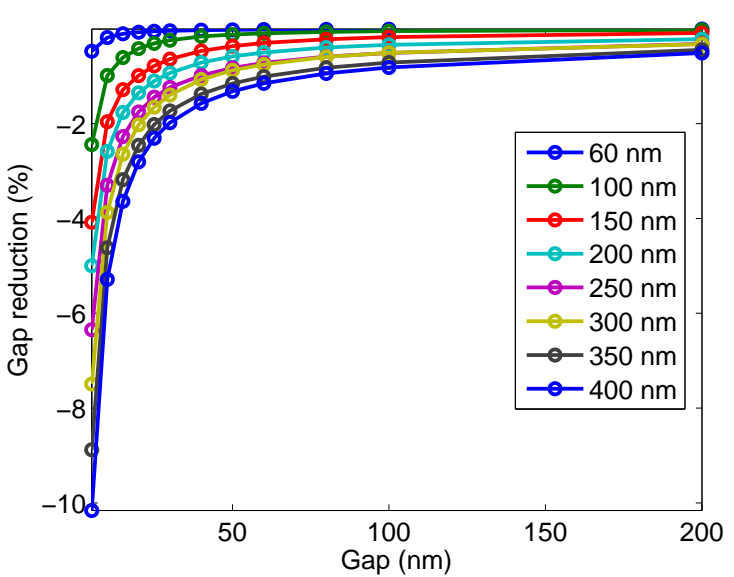

Figure 6: Gap reduction after illumination in percents as a function of the initial gap (in $\mathrm{nm}$ ).

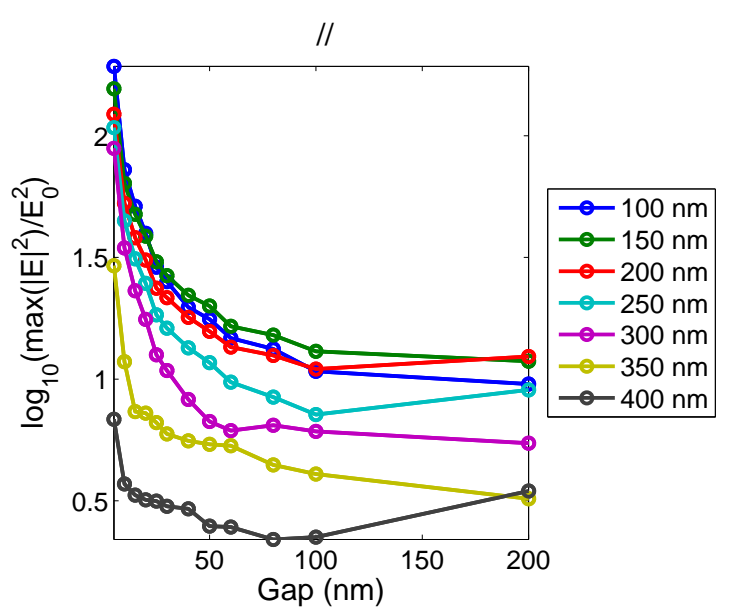

Figure 7: Intensity enhancement in the gap between the two nanorods.

to the maximum of the illumination intensity) is plotted in Fig. 7 in logscale. This local enhancement can be used to excite molecules for SERS biosensor. But the optimization of such biosensor should take into account the decrease of the gap as well as the increase of the temperature around the nanonantenna. Actually, the relative reduction of the gap is linearly related to the field enhancement $\eta=|E| /\left|E_{0}\right|$ (Fig. 8). This behavior will be specifically investigated in further studies as it could help to design the nanonantenna for specific applications. The same study for a transverse polarization of light is also investigated in the following.

\subsection{Polarization $\perp$ vs polarization $\|$}

It is well known that the polarization state of the illumination plays a key role in the nanoworld [30, 31, 4]. The electromagnetic field around dimer has not the same behavior if the polarization of the incoming light is upright to the nanonantenna axis. In particular, the field in the gap is weaker. We investigate the influence of this polarization of the incoming illumination on the dilation, of the gap and of 


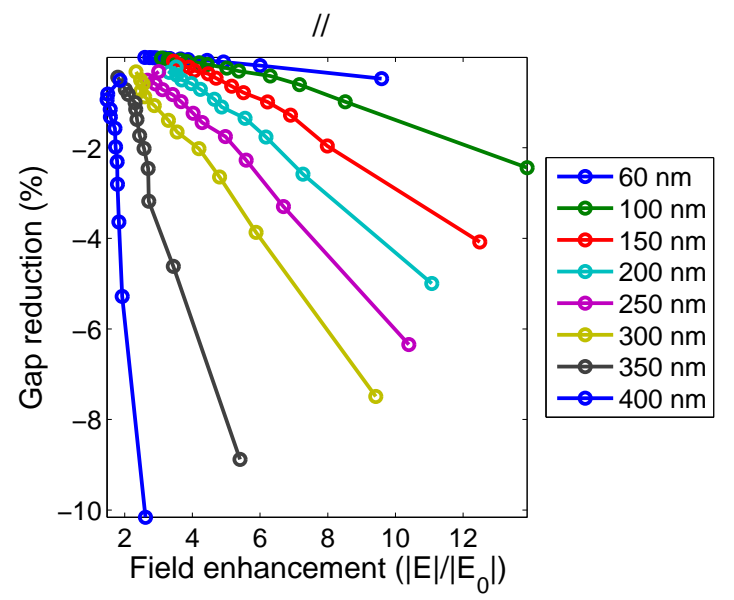

Figure 8: Linear behavior of the relative gap reduction as a function of the field enhancement.

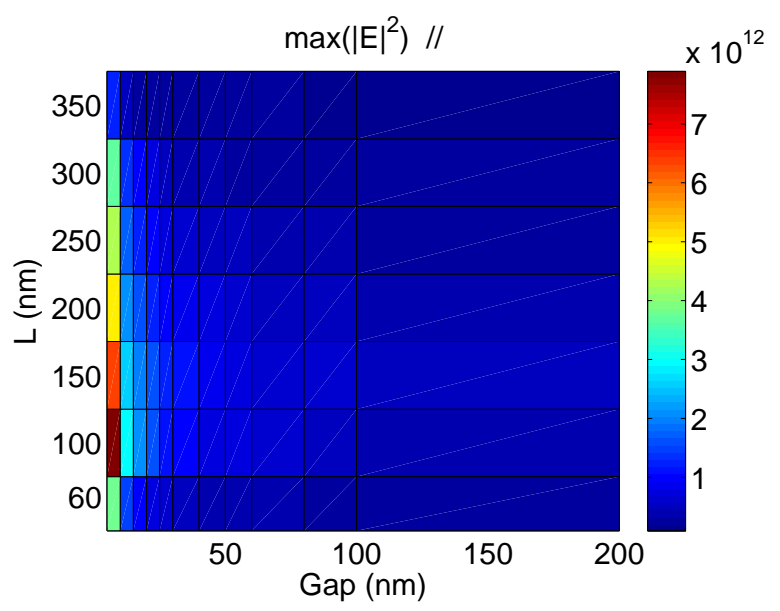

Figure 9: Maximum of intensity $|E|^{2},\left(V^{2} / m^{2}\right)$, as a function of the particle length $L$ and the gap for parallel polarization.

the antenna length. For comparison, the plots of the temperature elevation, the gap reduction and the temperature elevation are given for parallel and upright to the nanonatenna axis polarizations

The field enhancement in upright polarization is weaker than in parallel and the influence of the gap is less than that of the length (Figs 9 and 10). The contrast of the intensity is also decreased in the map 10. The gap is no more governing the field enhancement.

The same behavior can be observed in the temperature elevation (Figs 11 and 12). Whatever the gap is, the temperature depends mainly on the length of the nanonatenna. Nevertheless, the magnitude of the maximum of temperature elevation is almost the same in both cases.

Figures 13 and 14 are more correlated. The maximum of the gap reduction occurs for a small gap and for large antenna lengthes. The gap is more reduced with upright polarization.

Surprisingly, the global dilation of the gap exhibits the

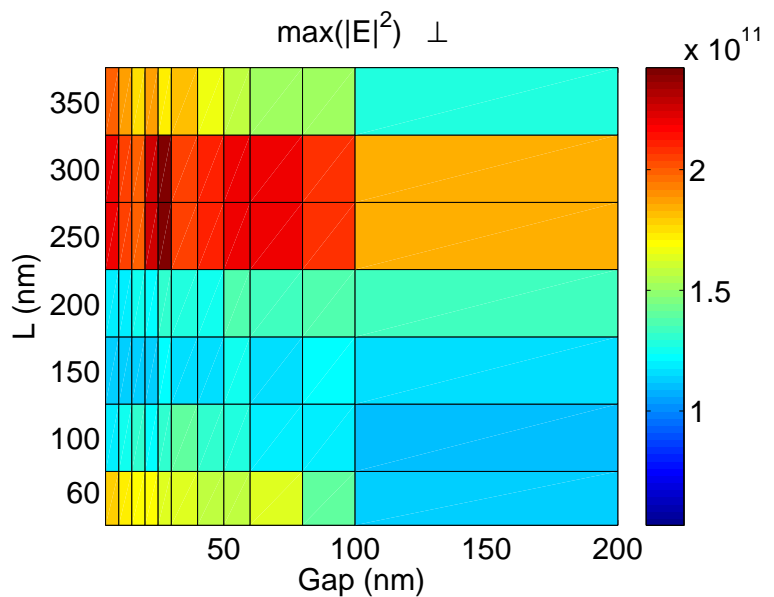

Figure 10: Maximum of intensity $|E|^{2},\left(V^{2} / m^{2}\right)$, as a function of the particle length $L$ and the gap for perpendicular polarization

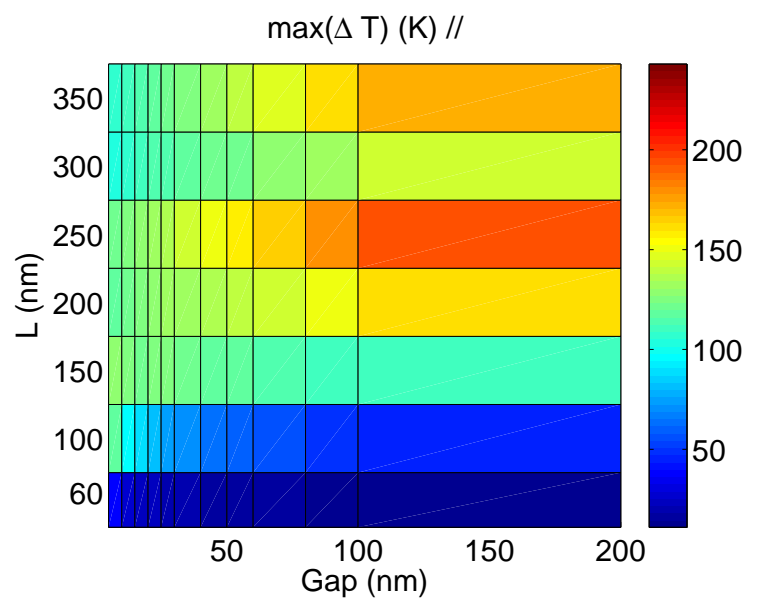

Figure 11: Maximum of elevation of temperature, $(K)$, as a function of the particle length $L$ and the gap for parallel polarization.

same behavior for both polarizations, as a function of the length and the gap. Moreover the effect of the field enhancement in the gap for parallel polarization seems to have less influence on temperature and dilation, excepted for the shortest gap, for which the scattering of this nanosource depresses the absorbed energy required for the temperature elevation.

\subsection{From 2D to $3 \mathrm{D}$ problems ?}

The 2D approach has been chosen for its speed of computation with the objective to optimize such nanostructures for biological applications [32, 33] or to retrieve unknown experimental data from measurements [34]. Nevertheless, the order of magnitude of the temperature and dilation could differ, if computed for 2D or 3D geometries. The order of magnitude of the 2D results should be faced to these obtained from 3D computation.

The electromagnetic field absorbed in a sphere or an 


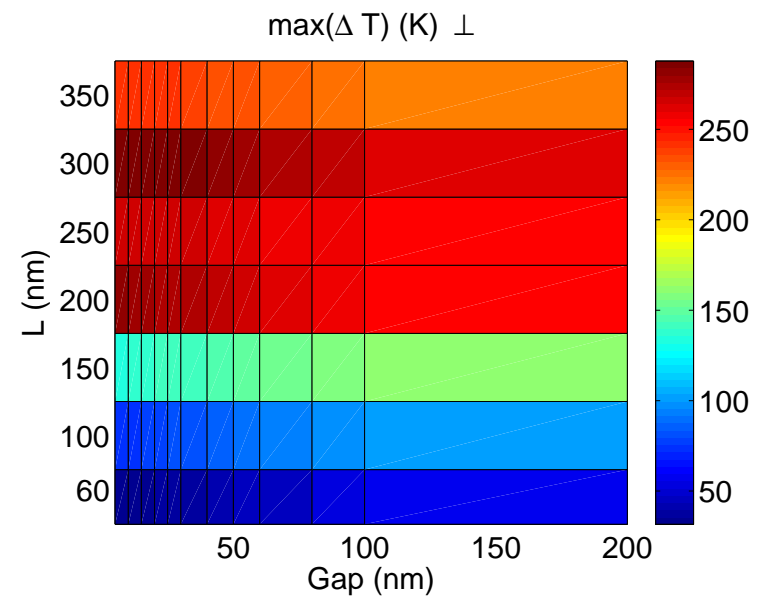

Figure 12: Maximum of elevation of temperature, $(K)$, as a function of the particle length $L$ and the gap for perpendicular polarization

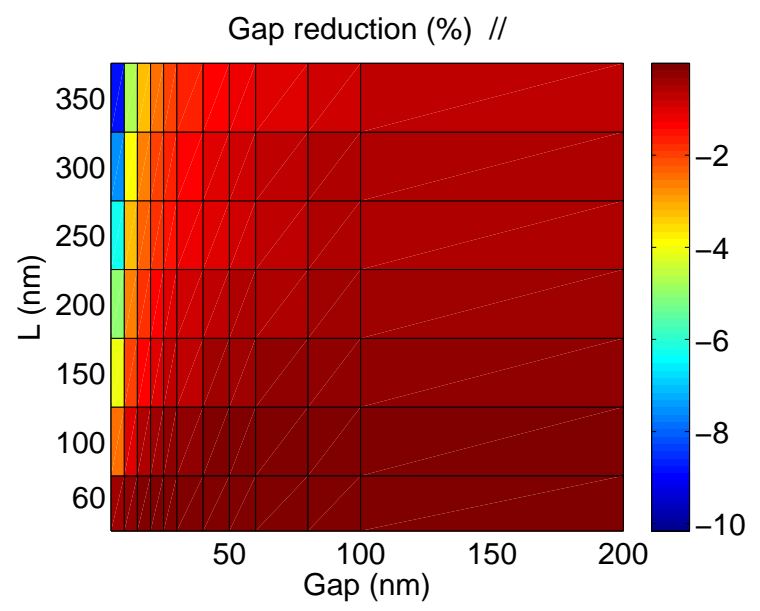

Figure 13: Relative reduction of the gap, (\%), as a function of the particle length $L$ and the gap for parallel polarization.

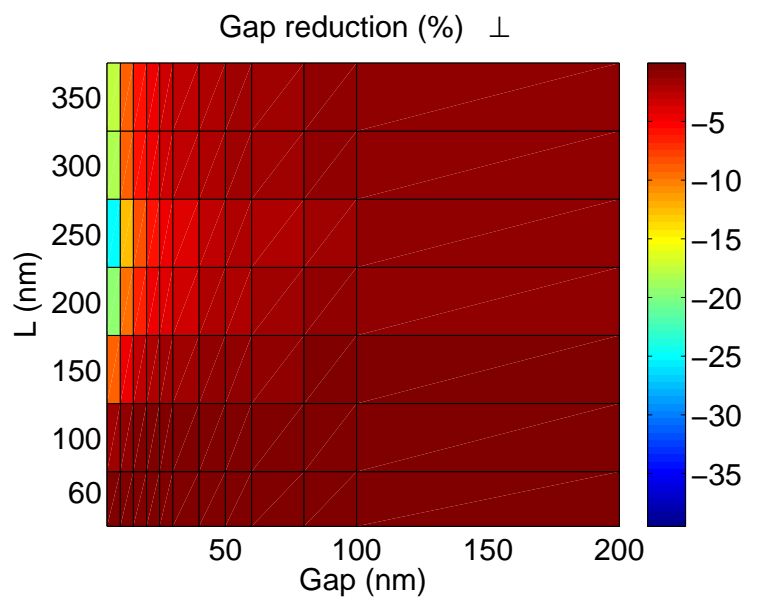

Figure 14: Relative reduction of the gap, (\%), as a function of the particle length $L$ and the gap for perpendicular polarization

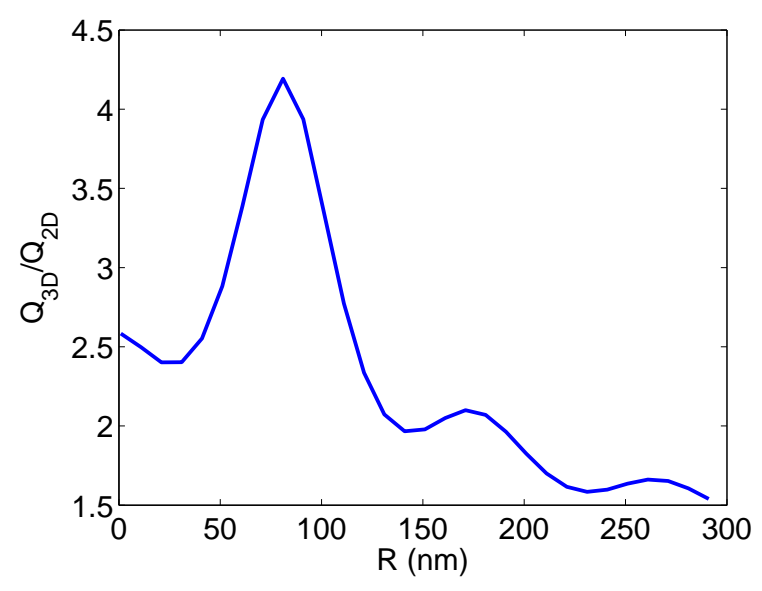

Figure 15: Ratio of the mean value of $Q$ for the sphere and the cylinder: $Q_{3 D} / Q_{2 D}$ as a function of the radius of the objects.

infinite cylinder of same radius can be analytically calculated [35]. For simplicity, we consider the same material parameters as in the previous sections. The mean value of the source term $Q$ for the stationary heat equation (Eq. 5) can therefore be evaluated and then, solving the stationary heat equation in the appropriate system of coordinates gives the temperature elevation:

$$
\begin{aligned}
& T_{2 D}(0) \approx \frac{Q_{3 D}}{6 k_{2}} R^{2} \\
& T_{3 D}(0) \approx \frac{Q_{2 D}}{4 k_{2}} R^{2}
\end{aligned}
$$

and the ratio of the temperature elevations:

$$
\frac{T_{3 D}(0)}{T_{2 D}(0)} \approx \frac{1}{3} \frac{Q_{3 D}}{Q_{2 D}}
$$

The ratio of their mean value $Q_{3 D} / Q_{2 D}$ is about 3 for a radius between 40 and $70 \mathrm{~nm}$, the figure 15 . The curve is oscillating between 1.5 and 4.2. Consequently, the ratio of the source terms offsets partially the coefficient $1 / 3$ but the ratio tends torward $1 / 2$ for radii larger than $200 \mathrm{~nm}$. In the case of $R=100 \mathrm{~nm}$, the temperature elevation is about the same for the two nano-objects. The figure 16 shows the finite element computation of the temperature for a cylinder and a sphere of radius $100 \mathrm{~nm}$. The finite element gives satisfactory results, especially in the metal. In this case, the maximal deformation is about $2 \mathrm{~nm}$.

The 2D finite element approach gives therefore the correct order of magnitude for the temperature, and the dilation can be quickly computed from this temperature elevation. Nevertheless, the physical constants of gold are not well known at this scale. In particular, the relative permittivity and the thermal conductivity could vary by a factor greater than $2[36]$. 


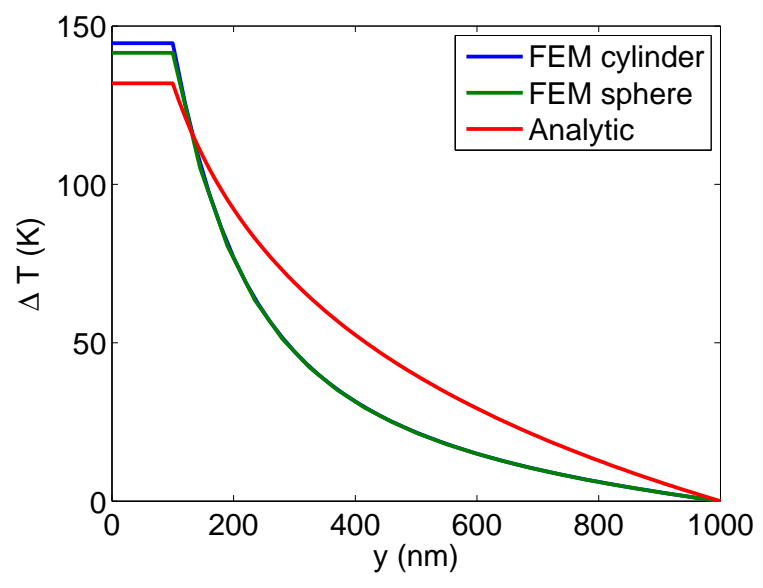

Figure 16: Temperature elevation along $y$ axis for a sphere and an infinite cylinder along $x$ direction. Finite element and analytical results for the sphere are compared.

\section{Conclusions}

Two complementary numerical approaches of the photo induced dilations in nanostructures are proposed. The numerical values of temperature and dilation are characteristics of the 2D objects and therefore the method should be extended to 3D nanonantenna. Nevertheless, the multiphysics approaches, with mesh deformation induced by photo-thermal dilation, could help to improve numerical methods especially for non linear processes. Actually, the sequential approach seems to be the equivalent to the coupled approach for linear problems, where physical constants do not dependent on the temperature. The non linear or strongly coupled models are an open domain for numerical approaches and finite element method will certainly be an efficient tool to solve them. The reliability of results depends on the measurement of optical, thermal and mechanical constants for gold and therefore sensitivity and tolerance studies [37, 38, 39] should be done before the inverse problem resolution $[34,40]$. The next step could consist in tuning theoretically the temperature around nanostructures with optimization methods [34, 38], to design specific samples that could be characterized experimentally.

\section{Acknowledgement}

This work was supported by the "Conseil Régional de Champagne Ardenne", the "Conseil Général de l'Aube" and the Nanoantenna European Project (FP7 Health-F52009-241818).

\section{References}

[1] A. Ambrosioa, M. Allegrini, G. Latini, and F. Cacialli, "Thermal processes in metal-coated fiber probes for near-field experiments," Appl. Phys. Lett., vol. 86, p. 203109, 2005.
[2] A. Kittel, W. Müller-Hirsch, J. Parisi, S. Biehs, and D. Reddig, "Near-field heat transfer in a scanning thermal microscope," Phys. Rev. Lett., vol. 95, p. 224301, 2005.

[3] P. Gucciardi, S. Paranè, A. Ambrosio, M. Allegrini, A. Downes, G. Latini, O. Fenwick, and F. Cacialli, "Observation of tip-to-sample heat transfer in near-field optical microscopy using metal-coated fiber probes," Appl. Phys. Lett., vol. 86, p. 203109, 2005.

[4] C. Baffou, M. Kreuzer, F. Kulzer, and R. Quidant, "Temperature mapping near plasmonic nansotructures using fluorescence polarization anistropy," Opt. Express, vol. 17, no. 5, pp. 3291-3298, 2009.

[5] A. L. Rosa, B. Yakobson, and H. Hallena, "Origins and effects of thermal processes on near-field optical probes," Appl. Phys. Lett., vol. 67, no. 18, pp. 25972599, 1995.

[6] J. Wang, Z. Shen, B. Xu, X. Ni, J. Guan, and J. Lu, "Simulation on thermoelastic stress field and laser ultrasound waveform in non-metallic materials by using fem," Appl. Phys. A, vol. 84, pp. 301-307, 2006.

[7] P. Gucciardi, M. Colloci, M. Labardi, and M. Allegrini, "Thermal-expansion effects in near-field optical microscopy fiber probes induced by laser light absorption," Appl. Phys. Lett., vol. 75, pp. 3408-3410, 1999.

[8] A. H. L. Rosa and H. D. Hallen, "Compact method for optical induction of proximal probe heating and elongation," Appl. Opt., vol. 41, pp. 2015-2019, 2002.

[9] T. Grosges, S. Petit, D. Barchiesi, and S. Hudlet, "Numerical modeling of the subwavelength phase-change recording using an apertureless scanning near-field optical microscope," Opt. Express, vol. 12, pp. 59875995, 2004.

[10] T. Pagnot, D. Barchiesi, D. van Labeke, and C. Pieralli, "Use of SNOM architecture to study fluorescence and energy transfer near a metal," Opt. Lett., vol. 22, no. 2, pp. 120-122, 1997.

[11] G. Parent, D. Van Labeke, and D. Barchiesi, "Fluorescence lifetime of a molecule near a corrugated interface. application to near-field microscopy," J. Opt. Soc. Am. A, vol. 16, pp. 896-908, 1999.

[12] J.-S. Huang, J. Kern, P. Geisler, P. Weinmann, M. Kamp, A. Forchel, P. Biagioni, and B. Hecht, "Mode imaging and selection in strongly coupled nanoantennas," NanoLetters, vol. 10, no. 6, pp. 2105$2110,2010$.

[13] H. Fischer and O. J. F. Martin, "Engineering the optical response of plasmonic nanoantennas," Opt. Express, vol. 16, no. 12, pp. 9144-9154, 2008. 
[14] L. Yang, C. Du, and X. Luo, "Numerical study of optical properties of single silver nanobowtie with anisotropic topology," Appl. Phys. B, vol. 92, pp. 53$59,2008$.

[15] N. Guillot, , H. Shen, B. Frémaux, O. Péron, E. Rinnert, T. Toury, and M. Lamy de la Chapelle, "Surface enhanced Raman scattering optimization of gold nanocylinder arrays: Influence of the localized surface plasmon resonance and excitation wavelength," Appl. Phys. Lett., vol. 97, no. 2, pp. 023 113-023 116,, 2010.

[16] P. Guillemet and J. P. Bardon, "Conduction de la chaleur aux temps courts : les limites spatiotemporelles des modèles parabolique et hyperbolique," Int. J. Therm. Sci., vol. 39, pp. 968-982, 2000

[17] R. Bachelot, A. Fares, R. Fikri, D. Barchiesi, G. Lerondel, and P. Royer, "Coupling semiconductor lasers into single-mode optical fibers by use of tips grown by photopolymerization," Optics Letters, vol. 29, no. 17, pp. 1971-1973, 2004.

[18] T. Pagnot, D. Barchiesi, D. V. Labeke, and C. Pieralli, "Use of a SNOM architecture to study fluorescence and energy transfer near a metal," Opt. Lett., vol. 22, pp. 120-122, 1997.

[19] H. Borouchaki, T. Grosges, and D. Barchiesi, "Improved $3 \mathrm{~d}$ adaptive remeshing scheme applied in high electromagnetic field gradient computation," Finite Elements in Analysis and Design, vol. 46, no. 1-2, pp. 84-95, 2010.

[20] T. Grosges, H. Borouchaki, and D. Barchiesi, "Three dimensional adaptive meshing scheme applied to the control of the spatial representation of complex field pattern in electromagnetics," Appl. Phys. B - Lasers Opt., vol. 101, pp. 883-889, 2010.

[21] J. Jin, The Finite Element Method in Electromagnetics. New York: John Wiley and Sons, 1993.

[22] G. P. Agrawal and D. N. Pattanayak, "Gaussian beam propagation beyond the paraxial approximation," $J$. Opt. Soc. Am., vol. 69, no. 4, pp. 575-578, 1979.

[23] D. Barchiesi, T. Grosges, E. Kremer, and M. Lamy de la Chapelle, "Electromagnetic heat-induced in mesostructures: Computation of temperature in metallic dimers," PIERS Online, vol. 7, no. 5, pp. 406-410, 2011.

[24] D. Kazantseva, G. Guttroff, M. Bayer, and A. Forche, "Sample temperature measurement in a scanning near-field optical microscope," Appl. Phys. Lett., vol. 72, no. 6, pp. 689-691, 1998.

[25] Y. S. Ju, "Impact of nonequilibrium between electrons and phonons on heat transferin metallic nanoparticles suspended in dielectric media," J. Heat Transfer, vol. 127, no. 12, pp. 1400-1402, 2005.

[26] P. E. Hopkins, P. M. Norris, L. M. Phinney, S. A. Policastro, and R. G. Kelly, "Thermal conductivity in nanoporous gold films during electron-phonon nonequilibrium," J. Nanomaterials, vol. 2008, pp. $418050-1-7,2008$.

[27] R. F. Remis, "An effective inversion method based on the padé via lanczos process," in Progress In Electromagnetics Research Symposium, Cambridge, USA, March 2006, pp. 206-209.

[28] P. Vinsome, "Orthomin, an iterative method for solving sparse sets of simultaneous linear equations," in SPE Symposium on Numerical Simulation of Reservoir Performance. Los Angeles, California, USA: Society of Petroleum Engineers, February 1976, pp. 5729-MS 1-11.

[29] T. Grosges, H. Borouchaki, and D. Barchiesi, "Improved scheme for accurate computation of high electric near-field gradients," Opt. Express, vol. 15, no. 3, pp. 1307-1321, 2007.

[30] A. Madrazo, R. Carminati, M. Nieto-Vesperinas, and J.-J. Greffet, "Polarization effects in the optical interaction between a nanoparticle and a corrugated surface: Implications for apertureless near-field microscopy," J. Opt. Soc. Am. A, vol. 15, no. 1, pp. 109119, 1998.

[31] R. Bachelot, F. HDhili, D. Barchiesi, G. Lerondel, R. Fikri, P. Royer, N. Landraud, J. Peretti, F. Chaput, G. Lampel, J.-P. Boilot, and K. Lahlil, "Apertureless near-field optical microscopy: A study of the local tip field enhancement using photosensitive azobenzenecontaining films," Journal of Applied Physics, vol. 94, no. 3, pp. 2060-2072, 2003.

[32] T. Grosges, D. Barchiesi, T. Toury, and G. Gréhan, "Design of nanostructures for imaging and biomedical applications by plasmonic optimization," Opt. Lett., vol. 33, no. 23, pp. 2812-2814, 2008.

[33] S. Kessentini and D. Barchiesi, "A new strategy to improve particle swarm optimization exploration ability," in Intelligent Systems (GCIS), 2010 Second WRI Global Congress on, vol. 1. IEEE, 2010, pp. 27-30.

[34] D. Macías, A. Vial, and D. Barchiesi, "Application of evolution strategies for the solution of an inverse problem in Near-Field Optics," J. Opt. Soc. Am. A, vol. 21, no. 8, pp. $1465-1471,2004$.

[35] C. F. Bohren and D. R. Huffman, Absorption and Scattering of Light by Small Particles. New York: John Wiley \& Sons, Inc., 1998. 
[36] T. Laroche, A. Vial, and M. Roussey, "Crystalline structure's influence on the near-field optical properties of single plasmonic nanowires," Appl. Phys. Lett., vol. 91, pp. 123 101-1-123 101-3, 2007.

[37] D. Barchiesi, D. S. Kessentini, and T. Grosges, "Sensitivity analysis for designing active particles in photothermal cancer therapy," in Advances in Safety, Reliability and Risk Management, C. Bérenguer and A. Grall, Eds. London: Taylor \& Francis, 2011, pp. 2197-2204.

[38] T. Grosges, D. Barchiesi, S. Kessentini, G. Gréhan, and M. Lamy de la Chapelle, "Nanoshells for photothermal therapy: A monte-carlo based numerical study of their design tolerance," Biomedical $O p$ tics Express, vol. 2, no. 6, pp. 1584-1596, 2011, http://www.opticsinfobase.org/boe/abstract.cfm?URI=boe2-6-1584.

[39] D. Barchiesi, N. Lidgi-Guigui, and M. Lamy de la Chapelle, "Functionalization layer influence on the sensitivity of surface plasmon resonance (SPR) biosensor," Optics Communications, vol. 285, no. 6, pp. 1619-1623, 2012.

[40] D. Barchiesi, "Numerical retrieval of thin aluminium layer properties from SPR experimental data," Opt. Express, vol. 20, no. 8, pp. 9064-9078, 2012. 